\title{
The Modified Trapezoidal Rule for Computing Hypersingular Integral on Interval
}

\author{
Jin $\mathrm{Li}^{1,2}$ and Xiuzhen $\mathrm{Li}^{1}$ \\ ${ }^{1}$ School of Science, Shandong Jianzhu University, Jinan 25010, China \\ ${ }^{2}$ School of Mathematics, Shandong University, Jinan 250100, China \\ Correspondence should be addressed to Jin Li; lijin@lsec.cc.ac.cn
}

Received 3 August 2013; Revised 12 October 2013; Accepted 12 October 2013

Academic Editor: Kazutake Komori

Copyright (C) $2013 \mathrm{~J}$. Li and X. Li. This is an open access article distributed under the Creative Commons Attribution License, which permits unrestricted use, distribution, and reproduction in any medium, provided the original work is properly cited.

\begin{abstract}
The modified trapezoidal rule for the computation of hypersingular integrals in boundary element methods is discussed. When the special function of the error functional equals zero, the convergence rate is one order higher than the general case. A new quadrature rule is presented and the asymptotic expansion of error function is obtained. Based on the error expansion, not only do we obtain a high order of accuracy, but also a posteriori error estimate is conveniently derived. Some numerical results are also reported to confirm the theoretical results and show the efficiency of the algorithms.
\end{abstract}

\section{Introduction}

Consider the following integral:

$$
I(f, s):=f_{a}^{b} \frac{f(x)}{(x-s)^{p+1}} d x, \quad s \in(a, b), p=1,2,
$$

where $f_{a}^{b}$ denotes a Hadamard finite-part integral $(p=$ 1 is called hypersingular integral and $p=2$ is called supersingular integral) and $s$ is the singular point. The formulation of these classes of boundary value problems in terms of hypersingular integral equations has drawn lots of interest. Many scientific and engineering problems, such as acoustics, electromagnetic scattering, and fracture mechanics, can be reduced to boundary integral equations with hypersingular kernels. There exist several definitions, equivalent mathematically, for such kind of integrals in some literatures.

We mention the following one:

$$
f_{a}^{b} \frac{f(x)}{(x-s)^{2}} d x
$$

$$
\begin{aligned}
=\lim _{\varepsilon \rightarrow 0}\left\{\int_{a}^{s-\varepsilon} \frac{f(x)}{(x-s)^{2}} d x\right. \\
\left.\quad+\int_{s+\varepsilon}^{b} \frac{f(x)}{(x-s)^{2}} d x-\frac{2 f(s)}{\varepsilon}\right\}, \quad s \in(a, b) .
\end{aligned}
$$

Accurate calculation of boundary element methods (BEM) arising in boundary integral equations has been a subject of intensive research in recent years. The hypersingular integrals have certain properties different from regular and weak singular integrals. One of the major problems arising from boundary element method, for solving such integral equations, is how to evaluate the hypersingular integrals on the interval or on the circle efficiently.

Hypersingular integral must be considered in Hadamard finite-part sense. Numerous works [1-18] have been devoted towards developing efficient quadrature formulas. In 1983, the series expansion of hypersingular integral kernel on circle was firstly suggested by $\mathrm{Yu}$ [19]. He solved the harmonic and biharmonic natural boundary integral equations successfully. The Newton-Cotes methods to compute the hypersingular integral on interval were firstly studied by Linz [20] with generalized trapezoidal and Simpson rules which fail altogether when the singular point $s$ is close to a mesh point. In order to 
make the mesh be selected in such a way that $s$ falls near the center of a subinterval, two shorter subintervals at the end of the interval were allowed. Then Yu [21] gave new quadrature formulae to compute the case of singular point coinciding with the mesh point which presented that the error estimate is $O(h|\ln h|)$. In 1999, Wu and Yu [22] presented simple, easy to be implemented methods not affected by the location of singular point with calculation of double. In recent years, the case of singular point coincided with the mesh point, and $\mathrm{Wu}$ et al. [23] presented a modified trapezoidal rule and proved the $O(h)$ convergence rate.

In this paper, for the case of singular point coinciding with the mesh point a new quadrature rule is introduced. Based on the expansion of the error functional, the error estimate is presented and a posteriori error estimate is given. Then not only do we obtain a high order of accuracy, but also a posteriori error estimate is conveniently derived.

The rest of this paper is organized as follows. In Section 2, after introducing some basic formulas of the general (composite) trapezoidal rule and notations, we present our main result. In Section 3, the corresponding theoretical analysis is given. Finally, several numerical examples are given to validate our analysis.

\section{Main Result}

Let $a=x_{0}<x_{1}<\cdots<x_{n-1}<x_{n}=b$ be a uniform partition of the interval $[a, b]$ with mesh size $h=(b-a) / n$ and set

$$
x_{0 i}=x_{i-1}+\frac{h}{6}, \quad i=1,2, \ldots, n
$$

then we get the new partition:

$$
a=x_{00}<x_{01}<\cdots<x_{0 n}<x_{0, n+1}=b .
$$

We define $f_{L}(x)$, the modified trapezoidal interpolation for $f(x)$, as

$$
\begin{array}{r}
f_{L}(x)=\frac{x-x_{0 i}}{x_{0, i+1}-x_{0 i}} f\left(x_{0, i+1}\right)+\frac{x_{0, i+1}-x}{x_{0, i+1}-x_{0 i}} f\left(x_{0 i}\right), \\
x \in\left[x_{0 i}, x_{0, i+1}\right], 0 \leq i \leq n,
\end{array}
$$

and a linear transformation

$$
\begin{array}{r}
x=\widehat{x}_{0 i}(\tau):=(\tau+1) \frac{x_{0, i+1}-x_{0 i}}{2}+x_{0 i}, \\
i=1, \ldots, n-1, \tau \in[-1,1],
\end{array}
$$

from the reference element $[-1,1]$ to the subinterval $\left[x_{0 i}, x_{0, i+1}\right]$. For the two subintervals $\left[a, x_{01}\right]$ and $\left[x_{0 n}, b\right]$ near the end of the interval $[a, b], \tau$ values in $[2 / 3,1]$ and $[-1,2 / 3]$, respectively.

Replacing $f(x)$ in (1) with $f_{L}(x)$ gives the new composite trapezoidal rule:

$$
\begin{aligned}
I_{n}(f, s) & :=f_{a}^{b} \frac{f_{L}(x)}{(x-s)^{2}} d x=\sum_{i=0}^{n+1} \omega_{j}(s) f\left(x_{0 i}\right) \\
& =I(f, s)-E_{n}(f),
\end{aligned}
$$

where $\omega_{i}(s)$ is the Cotes coefficients:

$$
\begin{aligned}
\omega_{i}(s)= & \frac{1-\delta_{i 0}}{x_{0, i}-x_{0, i-1}} \ln \left|\frac{x_{0 i}-s}{x_{0, i-1}-s}\right| \\
& -\frac{1-\delta_{i, n+1}}{x_{0, i+1}-x_{0 i}} \ln \left|\frac{x_{0, i+1}-s}{x_{0 i}-s}\right|+\frac{\delta_{i 0}}{x_{00}-s}+\frac{\delta_{i, n+1}}{s-x_{0, n+1}},
\end{aligned}
$$

$0 \leq i \leq n+1, \delta_{i j}$ denotes the Kronecker delta, and $E_{n}(f)$ denotes the error functional.

Theorem 1. Assume $f(x) \in C^{1+\alpha}[a, b], \alpha \in[0,1)$. For the trapezoidal rule $I_{n}(f, s)$ defined in $(7)$, there exists a positive constant $C$, independent of $h$ and s, such that

$$
\left|E_{n}(f)\right| \leq C(|\ln h|+|\ln \gamma(\tau)|) h^{\alpha},
$$

where

$$
\gamma(\tau)=\min _{0 \leq i \leq n+1} \frac{\left|s-x_{i}\right|}{h}=\frac{1-|\tau|}{2}, \quad \tau \in(-1,1) .
$$

Proof. Let $R(x)=f(x)-f_{L}(x)$; then we have $|R(x)| \leq C h^{1+\alpha}$, as

$$
\begin{aligned}
I(f, s)-I_{n}(f, s)= & f_{a}^{b} \frac{f(x)-f_{L}(x)}{(x-s)^{2}} d x \\
= & f_{x_{0 m}}^{x_{0, m+1}} \frac{f(x)-f_{L}(x)}{(x-s)^{2}} d x \\
& +\sum_{i=0, i \neq m}^{n} \int_{x_{0 i}}^{x_{0, i+1}} \frac{f(x)-f_{L}(x)}{(x-s)^{2}} d x .
\end{aligned}
$$

For the first part of (11), since $R(x) \in C^{1+\alpha}[a, b]$, by Taylor expansion, we have

$$
|R(x)| \leq C h^{1+\alpha-i}, \quad i=0,1,2 .
$$

By the definition of finite-part integral,

$$
\begin{aligned}
f_{a}^{b} \frac{f(x)}{(x-s)^{2}} d x= & \frac{(b-a) f(s)}{(s-a)(b-s)}+f^{\prime}(s) \ln \frac{b-s}{s-a} \\
& +\int_{a}^{b} \frac{f(x)-f(s)-f^{\prime}(s)(x-s)}{(x-s)^{2}} d x,
\end{aligned}
$$

we have

$$
\begin{aligned}
f_{x_{0 m}}^{x_{0, m+1}} & \frac{R(x)}{(x-s)^{2}} d x \\
= & \frac{h R(s)}{\left(s-x_{0 m}\right)\left(x_{0, m+1}-s\right)}+R^{\prime}(s) \ln \frac{x_{0, m+1}-s}{s-x_{0 m}} \\
& +\int_{x_{0 m}}^{x_{0, m+1}} \frac{R(x)-R(s)-R^{\prime}(s)(x-s)}{(x-s)^{2}} d x .
\end{aligned}
$$


Now, we estimate the right hand side of (14) term by term. Since $R\left(x_{0 m}\right)=0$, we have

$$
\begin{aligned}
&\left|\frac{h R(s)}{\left(s-x_{0 m}\right)\left(x_{0, m+1}-s\right)}\right|=\left|\frac{h\left[R(s)-R\left(x_{0 m}\right)\right]}{\left(s-x_{0 m}\right)\left(x_{0, m+1}-s\right)}\right| \\
&=\left|\frac{h R_{m}^{\prime}\left(\xi_{m}\right)}{\left(s-x_{0, m+1}\right)}\right| \\
& \leq C h^{\alpha}, \quad \xi_{m} \in\left(x_{0 m}, s\right), \\
&\left|R^{\prime}(s) \ln \frac{x_{0, m+1}-s}{s-x_{0 m}}\right| \leq C|\ln \gamma(\tau)| h^{\alpha}, \\
&\left|\int_{x_{0 m}}^{x_{0, m+1}} \frac{R(x)-R(s)-R^{\prime}(s)(x-s)}{(x-s)^{2}} d x\right| \leq C h^{\alpha}, \\
& \eta_{m} \in\left(x_{0 m}, x_{0, m+1}\right) .
\end{aligned}
$$

For the second part of (11),

$$
\begin{aligned}
& \left|\left(\int_{a}^{x_{01}}+\sum_{i=1, i \neq m}^{n-1} \int_{x_{0 i}}^{x_{0, i+1}}+\int_{x_{0 n}}^{b}\right) \frac{R(x)}{(x-s)^{2}} d x\right| \\
& \leq \mid\left(\int_{a}^{x_{01}}+\sum_{i=1, i \neq m-1, m, m+1}^{n-1} \int_{x_{0 i}}^{x_{0, i+1}}\right. \\
& \left.+\int_{x_{0 n}}^{b}\right) \frac{R(x)}{(x-s)^{2}} d x \mid \\
& +\left|\int_{x_{0, m-1}}^{x_{0, m}} \frac{R(x)}{(x-s)^{2}} d x+\int_{x_{0, m+1}}^{x_{0, m+2}} \frac{R(x)}{(x-s)^{2}} d x\right| \\
& \leq \mid\left(\int_{a}^{x_{01}}+\sum_{i=1, i \neq m-1, m, m+1}^{n-1} \int_{x_{0 i}}^{x_{0, i+1}}\right. \\
& \left.+\int_{x_{0 n}}^{b}\right) \frac{R(x)}{(x-s)^{2}} d x \\
& +\mid \int_{x_{0, m-1}}^{x_{0 m}} \frac{R(x)-R\left(x_{0 m}\right)}{(x-s)^{2}} d x \\
& +\int_{x_{0, m+1}}^{x_{0, m+2}} \frac{R(x)-R\left(x_{0, m+1}\right)}{(x-s)^{2}} d x \mid \\
& =\left|\left(\int_{a}^{x_{01}}+\sum_{i=1, i \neq m-1, m, m+1}^{n-1} \int_{x_{0 i}}^{x_{0, i+1}}+\int_{x_{0 n}}^{b}\right) \frac{R(x)}{(x-s)^{2}} d x\right| \\
& +\mid \int_{x_{0, m-1}}^{x_{0 m}} \frac{R^{\prime}\left(\xi_{0 m}\right)\left(x-x_{0 m}\right)}{(x-s)^{2}} d x \\
& +\int_{x_{0, m+1}}^{x_{0, m+2}} \frac{R^{\prime}\left(\xi_{0, m+1}\right)\left(x-x_{0, m+1}\right)}{(x-s)^{2}} d x \mid \\
& \leq C h^{1+\alpha}\left(\int_{a}^{x_{01}}+\sum_{i=1, i \neq m-1, m, m+1}^{n-1} \int_{x_{0 i}}^{x_{0, i+1}}\right.
\end{aligned}
$$

$$
\begin{gathered}
\left.+\int_{x_{0 n}}^{b}\right) \frac{1}{(x-s)^{2}} d x \\
+C h^{\alpha}\left(\int_{x_{0, m-1}}^{x_{0 m}} \frac{d x}{|x-s|}+\int_{x_{0, m+1}}^{x_{0, m+2}} \frac{d x}{|x-s|}\right) \\
\leq C[|\ln \gamma(\tau)|+|\ln h|] h^{\alpha} .
\end{gathered}
$$

Combining (14), (15), and (16) leads to (9) and the proof is completed.

Firstly, we set

$$
\phi_{1}(x)= \begin{cases}-\frac{1}{2} \int_{-1}^{1} \frac{\tau^{2}-1}{\tau-x} d \tau, & |x|<1, \\ -\frac{1}{2} \int_{-1}^{1} \frac{\tau^{2}-1}{\tau-x} d \tau, & |x|>1 ;\end{cases}
$$

then we have

$$
\phi_{1}^{\prime}(x)= \begin{cases}-\frac{1}{2} f_{-1}^{1} \frac{\tau^{2}-1}{(\tau-x)^{2}} d \tau, & |x|<1, \\ -\frac{1}{2} \int_{-1}^{1} \frac{\tau^{2}-1}{(\tau-x)^{2}} d \tau, & |x|>1 .\end{cases}
$$

By straight calculation, we get

$$
\phi_{1}^{\prime}(x)=x \log \frac{1+x}{1-x}-2 .
$$

Let $J:=(-\infty,-1) \bigcup(-1,1) \bigcup(1,+\infty)$ and the operator $W$ : $C(J) \rightarrow C(-1,1)$ be defined as

$$
\begin{array}{r}
W f(\tau):=f(\tau)+\sum_{i=1}^{\infty}[f(2 i+\tau)+f(-2 i+\tau)], \\
\tau \in(-1,1) .
\end{array}
$$

Obviously, the operator $W$ is linear operator. Then we set

$$
S_{1}(\tau)=W \phi_{1}^{\prime}(\tau)=\phi_{1}^{\prime}(\tau)+\sum_{i=1}^{\infty}\left[\phi_{1}^{\prime}(2 i+\tau)+\phi_{1}^{\prime}(-2 i+\tau)\right] .
$$

Now we present our main results below. The proof will be given in the next section.

Theorem 2. Assume $f(x) \in C^{3}[a, b]$. For the trapezoidal rule $I_{n}(f, s)$ defined in $(7)$, there exists a positive constant $C$, independent of $h$ and $s$, such that

$$
E_{n}(f)=\frac{f^{\prime \prime}(s) h}{2} S_{1}(\tau)+\mathscr{R}_{f}(s),
$$

where $s=x_{0 i}+(1+\tau) h / 2, i=1,2, \ldots, n$, and

$$
\left|\mathscr{R}_{f}(s)\right| \leq C(\eta(s)+|\ln h|+|\ln \gamma(\tau)|) h^{2}
$$

$(\gamma(\tau)$ defined as (10)) and

$$
\eta(s)=\max \left\{\frac{1}{s-a}, \frac{1}{b-s}\right\} .
$$




\section{Proof of Main Results}

3.1. Preliminaries. In the following section, $C$ denotes certain constant independent of $h$ and $s$, and its value varies with places.

Lemma 3. Assume that $f(x) \in C^{3}[a, b]$ and $f_{L}(x)$ are defined by (5); there holds

$$
\begin{aligned}
f(x)-f_{L}(x)= & \frac{f^{\prime \prime}(s)}{2}\left(x-x_{0, i+1}\right)\left(x-x_{0 i}\right) \\
& +\mathscr{R}_{i}^{1}(x)+\mathscr{R}_{i}^{2}(x),
\end{aligned}
$$

where

$$
\begin{gathered}
\mathscr{R}_{i}^{1}(x)=\frac{\left(x-x_{0, i+1}\right)\left(x-x_{0 i}\right)}{6\left(x_{0, i+1}-x_{0 i}\right)}\left[f^{(3)}\left(\xi_{0 i}\right)\left(x-x_{0, i+1}\right)^{2}\right. \\
\left.-f^{(3)}\left(\eta_{0 i}\right)\left(x-x_{0 i}\right)^{2}\right], \\
\mathscr{R}_{i}^{2}(x)=\frac{f^{(3)}\left(\beta_{0 i}\right)}{2}\left(x-x_{0, i+1}\right)\left(x-x_{0 i}\right)(x-s),
\end{gathered}
$$

$\xi_{0 i}, \eta_{0 i}, \beta_{0 i} \in\left(x_{0 i}, x_{0, i+1}\right)$, and

$$
\left|\mathscr{R}_{i}^{1}(x)\right| \leq C h^{3} .
$$

Proof. Taking Taylor expansion for $f\left(x_{0 i}\right), f\left(x_{0, i+1}\right)$ at $x$, there holds

$$
\begin{aligned}
f(x) & -f_{L}(x) \\
= & \frac{f^{\prime \prime}(x)}{2 !}\left(x-x_{0, i+1}\right)\left(x-x_{0 i}\right) \\
& +\frac{\left(x-x_{0, i+1}\right)\left(x-x_{0 i}\right)}{6\left(x_{0, i+1}-x_{0 i}\right)}\left[f^{(3)}\left(\xi_{0 i}\right)\left(x-x_{0, i+1}\right)^{2}\right. \\
& \left.-f^{(3)}\left(\eta_{0 i}\right)\left(x-x_{0 i}\right)^{2}\right] \\
:= & \frac{f^{\prime \prime}(s)}{2}\left(x-x_{0, i+1}\right)\left(x-x_{0 i}\right)+\mathscr{R}_{i}^{1}(x)+\mathscr{R}_{i}^{2}(x) ;
\end{aligned}
$$

by applying Taylor expansion to $f^{\prime \prime}(x)$ in (28) at $s$, we have

$$
f^{\prime \prime}(x)=f^{\prime \prime}(s)+f^{(3)}\left(\eta_{i}\right)(x-s) .
$$

Therefore, (25) can be obtained directly from (28) and (29).

Lemma 4. Assume that $s \in\left(x_{0 i}, x_{0, i+1}\right)$ and $c_{i}=2\left(s-x_{0 i}\right) / h-$ $1,1 \leq i \leq n-1$; there holds

$$
\phi_{1}^{\prime}\left(c_{i}\right)= \begin{cases}-\frac{1}{2 h} f_{x_{0 i}}^{x_{0, i+1}} \frac{\left(x-x_{0 i}\right)\left(x-x_{0, i+1}\right)}{(x-s)^{2}} d x, & i=m, \\ -\frac{1}{2 h} \int_{x_{0 i}}^{x_{0, i+1}} \frac{\left(x-x_{0 i}\right)\left(x-x_{0, i+1}\right)}{(x-s)^{2}} d x, & i \neq m .\end{cases}
$$

Proof. According to (2) and the linear transformation (6) for $i=m$, we have

$$
\begin{aligned}
& f_{x_{0 m}}^{x_{0, m+1}} \frac{\left(x-x_{0 m}\right)\left(x-x_{0, m+1}\right)}{(x-s)^{2}} d x \\
&=h \lim _{\varepsilon \rightarrow 0}\left\{\left(\int_{-1}^{c_{m}-(2 \varepsilon / h)}+\int_{c_{m}+(2 \varepsilon / h)}^{1}\right)\right. \\
&\left.\quad \times \frac{\tau^{2}-1}{\left(\tau-c_{m}\right)^{2}} d \tau-\frac{2\left(\tau^{2}-1\right)}{\varepsilon}\right\} \\
&=h f_{-1}^{1} \frac{\tau^{2}-1}{\left(\tau-c_{m}\right)^{2}} d \tau=-2 h \phi_{1}^{\prime}\left(c_{m}\right),
\end{aligned}
$$

where we have used $x=\widehat{x}_{0 m}(\tau)$. The second identity can be similarly obtained.

Lemma 5. Suppose $\phi^{\prime}(x)$ and $\eta(s)$ are defined by (18) and (24), respectively; then one has

$$
\left|\sum_{i=m}^{\infty} \phi_{1}^{\prime}(2 i+\tau)+\sum_{i=n-m}^{\infty} \phi_{1}^{\prime}(-2 i+\tau)\right| \leq C \eta(s) h,
$$

where $\sum^{\prime}$ denotes that the first interval is certain part of the reference element.

Proof. By straightforward calculation, we have

$$
\left|\phi_{1}^{\prime}(x)\right| \leq C \int_{-1}^{1} \frac{d \tau}{|\tau-x|^{2}} .
$$

Noting that $s=x_{0 m}+((\tau+1) / 2) h=a+(m+(\tau / 2)) h-(h / 3)$, we have $2(s-a) / h=3 \tau+6 m-2$ and

$$
\begin{aligned}
& \left|\sum_{i=m}^{\infty} \phi_{1}^{\prime}(2 i+\tau)\right| \\
& \quad \leq C\left[\int_{2 / 3}^{1} \frac{d t}{|2 i+\tau-t|^{2}}+\sum_{i=m+1}^{\infty} \int_{-1}^{1} \frac{d t}{|2 i+\tau-t|^{2}}\right] \\
& \quad=C \int_{3 \tau+6 m-2}^{\infty} \frac{d x}{x^{2}}=\frac{C}{3 \tau+6 m-2}=\frac{C h}{s-a} .
\end{aligned}
$$

On the other hand, since $b=a+n h$, we have $6(b-s) / h=$ $6(n-m)-(3 \tau-2)$ and

$$
\begin{aligned}
& \left|\sum_{i=n-m}^{\infty} \phi_{1}^{\prime}(\tau-2 i)\right| \\
& \quad \leq C\left[\int_{-1}^{2 / 3} \frac{d t}{|2 i-\tau+t|^{2}}+\sum_{i=n-m+1}^{\infty} \int_{-1}^{1} \frac{d t}{|2 i-\tau+t|^{2}}\right] \\
& \quad=C \int_{6(n-m)-(3 \tau-2)}^{\infty} \frac{d x}{x^{2}}=\frac{C}{6(n-m)-(3 \tau-2)} \\
& \quad=\frac{C h}{b-s} .
\end{aligned}
$$


Combining (34) and (35), we get (32).

Set

$$
H_{m}(x)=f(x)-f_{L}(x)-\frac{f^{\prime \prime}(s)}{2}\left(x-x_{0, m+1}\right)\left(x-x_{0 m}\right) .
$$

Lemma 6. Under the same assumptions of Theorem 2, for $H_{m}(x)$ in (36), there holds that

$$
\left|f_{x_{0 m}}^{x_{0, m+1}} \frac{H_{m}(x)}{(x-s)^{2}} d x\right| \leq C|\ln \gamma(\tau)| h^{2}
$$

where $\gamma(\tau)$ is defined in (10).

Proof. Since $f(x) \in C^{3}[a, b]$, by Taylor expansion, we have

$$
\left|H_{m}^{(i)}(x)\right| \leq C h^{3-i}, \quad i=0,1,2 .
$$

By the definition of finite-part integral (13), we have

$$
\begin{aligned}
f_{x_{0 m}}^{x_{0, m+1}} & \frac{H_{m}(x)}{(x-s)^{2}} d x \\
= & \frac{h H_{m}(s)}{\left(s-x_{0 m}\right)\left(x_{0, m+1}-s\right)}+H_{m}^{\prime}(s) \ln \frac{x_{0, m+1}-s}{s-x_{0 m}} \\
& \quad+\int_{x_{0 m}}^{x_{0, m+1}} \frac{H_{m}(x)-H_{m}(s)-H_{m}^{\prime}(s)(x-s)}{(x-s)^{2}} d x .
\end{aligned}
$$

Now, we estimate the right hand side of (39) term by term. Since $H_{m}\left(x_{0 m}\right)=0$, we have

$$
\begin{aligned}
& \left|\frac{h H_{m}(s)}{\left(s-x_{0 m}\right)\left(x_{0, m+1}-s\right)}\right|=\left|\frac{h\left[H_{m}(s)-H_{m}\left(x_{0 m}\right)\right]}{\left(s-x_{0 m}\right)\left(x_{0, m+1}-s\right)}\right| \\
& =\left|\frac{h H_{m}^{\prime}\left(\xi_{m}\right)}{\left(s-x_{0, m+1}\right)}\right| \\
& \leq C h^{2}, \quad \xi_{m} \in\left(s, x_{0, m+1}\right), \\
& \left|H_{m}^{\prime}(s) \ln \frac{x_{0, m+1}-s}{s-x_{0 m}}\right| \leq C|\ln \gamma(\tau)| h^{2}, \\
& \left|\int_{x_{0 m}}^{x_{0, m+1}} \frac{H_{m}(x)-H_{m}(s)-H_{m}^{\prime}(s)(x-s)}{(x-s)^{2}} d x\right| \\
& =\left|\int_{x_{0 m}}^{x_{0, m+1}} \frac{1}{2} H_{m}^{\prime \prime}\left(\eta_{m}\right) d x\right| \leq C h^{2}, \quad \eta_{m} \in\left(x_{0 m}, x_{0, m+1}\right) .
\end{aligned}
$$

Combining (40), (41), and (42) leads to (37) and the proof is completed.
Proof of Theorem 2. Consider

$$
\begin{aligned}
& \left(\int_{a}^{x_{0 m}}+\int_{x_{0, m+1}}^{b}\right) \frac{f(x)}{(x-s)^{2}} d x-\sum_{i=0, i \neq m}^{n} \int_{0 i}^{x_{0, i+1}} \frac{f_{L}(x)}{(x-s)^{2}} d x \\
& =\sum_{i=0, i \neq m}^{n-1} \int_{x_{0 i}}^{x_{0, i+1}} \frac{f(x)-f_{L}(x)}{(x-s)^{2}} d x \\
& =\frac{f^{\prime \prime}(s)}{2} \sum_{i=1, i \neq m}^{n-1} \int_{x_{0 i}}^{x_{0, i+1}} \frac{\left(x-x_{0 i}\right)\left(x-x_{0, i+1}\right)}{(x-s)^{2}} d x \\
& \quad+\sum_{i=0, i \neq m}^{n-1} \int_{x_{0 i}}^{x_{0, i+1}} \frac{\mathscr{R}_{i}^{1}(x)}{(x-s)^{2}} d x+\sum_{i=0, i \neq m}^{n-1} \int_{x_{0 i}}^{x_{0, i+1}} \frac{\mathscr{R}_{i}^{2}(x)}{(x-s)^{2}} d x \\
& \quad+\int_{a}^{x_{01}} \frac{f(x)-f_{L}(x)}{(x-s)^{2}} d x+\int_{x_{0 n}}^{b} \frac{f(x)-f_{L}(x)}{(x-s)^{2}} d x .
\end{aligned}
$$

By the definition of $E_{m}(x)$, we have

$$
\begin{aligned}
f_{x_{0 m}}^{x_{0, m+1}} & \frac{f(x)-f_{L}(x)}{(x-s)^{2}} d x \\
= & f_{x_{0 m}}^{x_{0, m+1}} \frac{\mathscr{H}_{m}(x)}{(x-s)^{2}} d x \\
& +\frac{f^{\prime \prime}(s)}{2} f_{x_{0, m}}^{x_{0, m+1}} \frac{\left(x-x_{0 i}\right)\left(x-x_{0, i+1}\right)}{(x-s)^{2}} d x .
\end{aligned}
$$

Putting (43) and (44) together, we have

$$
\begin{aligned}
f_{a}^{b} \frac{f(x)-f_{L}(x)}{(x-s)^{2}} d x= & \sum_{i=1}^{n-1} f_{x_{0 i}}^{x_{0, i+1}} \frac{f(x)-f_{L}(x)}{(x-s)^{2}} d x \\
& +\int_{a}^{x_{01}} \frac{f(x)-f_{L}(x)}{(x-s)^{2}} d x \\
& +\int_{x_{0 n}}^{b} \frac{f(x)-f_{L}(x)}{(x-s)^{2}} d x \\
= & -\frac{f^{\prime \prime}(s)}{2} h S_{1}(\tau)+\mathscr{R}_{f}(s),
\end{aligned}
$$

where

$$
\begin{gathered}
\mathscr{R}_{f}(s)=\mathscr{R}^{1}(s)+\mathscr{R}^{2}(s)+\mathscr{R}^{3}(s), \\
\mathscr{R}^{1}(s)=f_{x_{0 m}}^{x_{0, m+1}} \frac{\mathscr{H}_{m}(x)}{(x-s)^{2}} d x, \\
\mathscr{R}^{2}(s)=\left(\int_{a}^{x_{01}}+\sum_{i=1, i \neq m}^{n-1} \int_{x_{0 i}}^{x_{0, i+1}}+\int_{x_{0 n}}^{b}\right) \frac{\mathscr{R}_{i}^{1}(x)}{(x-s)^{2}} d x \\
+\left(\int_{a}^{x_{01}}+\sum_{i=1, i \neq m}^{n-1} \int_{x_{0 i}}^{x_{0, i+1}}+\int_{x_{0 n}}^{b}\right) \frac{\mathscr{R}_{i}^{2}(x)}{(x-s)^{2}} d x, \\
\mathscr{R}^{3}(s)=h \frac{f^{\prime \prime}(s)}{2}\left[\sum_{i=m}^{\infty} \phi_{1}^{\prime}(2 i+\tau)+\sum_{i=n-m}^{\infty} \phi_{1}^{\prime}(-2 i+\tau)\right] .
\end{gathered}
$$


For the first part of $\mathscr{R}^{2}(s)$,

$$
\begin{aligned}
& \left|\left(\int_{a}^{x_{01}}+\sum_{i=1, i \neq m}^{n-1} \int_{x_{0 i}}^{x_{0, i+1}}+\int_{x_{0 n}}^{b}\right) \frac{\mathscr{R}_{i}^{1}(x)}{(x-s)^{2}} d x\right| \\
& \leq\left|\left(\int_{a}^{x_{01}}+\sum_{i=1, i \neq m-1, m, m+1}^{n-1} \int_{x_{0 i}}^{x_{0, i+1}}+\int_{x_{0 n}}^{b}\right) \frac{\mathscr{R}_{i}^{1}(x)}{(x-s)^{2}} d x\right| \\
& +\left|\int_{x_{0, m-1}}^{x_{0, m}} \frac{\mathscr{R}_{m-1}^{1}(x)}{(x-s)^{2}} d x+\int_{x_{0, m+1}}^{x_{0, m+2}} \frac{\mathscr{R}_{m+1}^{1}(x)}{(x-s)^{2}} d x\right| \\
& \leq\left|\left(\int_{a}^{x_{01}}+\sum_{i=1, i \neq m-1, m, m+1}^{n-1} \int_{x_{0 i}}^{x_{0, i+1}}+\int_{x_{0 n}}^{b}\right) \frac{\mathscr{R}_{i}^{1}(x)}{(x-s)^{2}} d x\right| \\
& +\mid \int_{x_{0, m-1}}^{x_{0 m}} \frac{\mathscr{R}_{m-1}^{1}(x)-\mathscr{R}_{m-1}^{1}\left(x_{0 m}\right)}{(x-s)^{2}} d x \\
& +\int_{x_{0, m+1}}^{x_{0, m+2}} \frac{\mathscr{R}_{m+1}^{1}(x)-\mathscr{R}_{m+1}^{1}\left(x_{0, m+1}\right)}{(x-s)^{2}} d x \mid \\
& =\left|\left(\int_{a}^{x_{01}}+\sum_{i=1, i \neq m-1, m, m+1}^{n-1} \int_{x_{0 i}}^{x_{0, i+1}}+\int_{x_{0 n}}^{b}\right) \frac{\mathscr{R}_{i}^{1}(x)}{(x-s)^{2}} d x\right| \\
& +\mid \int_{x_{0, m-1}}^{x_{0 m}} \frac{\mathscr{R}_{m-1}^{1}{ }^{\prime}\left(\xi_{0 m}\right)\left(x-x_{0 m}\right)}{(x-s)^{2}} d x \\
& +\int_{x_{0, m+1}}^{x_{0, m+2}} \frac{\mathscr{R}_{m+1}^{1}{ }^{\prime}\left(\xi_{0, m+1}\right)\left(x-x_{0, m+1}\right)}{(x-s)^{2}} d x \\
& \leq \operatorname{Ch}^{3}\left(\int_{a}^{x_{01}}+\sum_{i=1, i \neq m-1, m, m+1}^{n-1} \int_{x_{0 i}}^{x_{0, i+1}}+\int_{x_{0 n}}^{b}\right) \frac{1}{(x-s)^{2}} d x \\
& +C^{2}\left(\int_{x_{0, m-1}}^{x_{0 m}} \frac{d x}{|x-s|}+\int_{x_{0, m+1}}^{x_{0, m+2}} \frac{d x}{|x-s|}\right) \\
& \leq C[|\ln \gamma(\tau)|+|\ln h|] h^{2} \text {, }
\end{aligned}
$$

where $\xi_{0 m} \in\left(x_{0, m-1}, x_{0 m}\right)$ and $\xi_{0, m+1} \in\left(x_{0, m}, x_{0, m+1}\right)$. We have also used the identity $\mathscr{R}_{m-1}^{1}\left(x_{0 m}\right)=\mathscr{R}_{m+1}^{1}\left(x_{0, m+1}\right)=0$, $\left|x-x_{0 m}\right|<|x-s|$, and $\left|x-x_{0, m+1}\right|<|x-s|$.

For the second part of $\mathscr{R}^{2}(s)$,

$$
\begin{aligned}
& \left|\left(\int_{a}^{x_{01}}+\sum_{i=1, i \neq m}^{n-1} \int_{x_{0 i}}^{x_{0, i+1}}+\int_{x_{0 n}}^{b}\right) \frac{\mathscr{R}_{i}^{2}(x)}{(x-s)^{2}} d x\right| \\
& \leq C^{2}\left(\int_{a}^{x_{01}}+\sum_{i=1, i \neq m}^{n-1} \int_{x_{0 i}}^{x_{0, i+1}}+\int_{x_{0 n}}^{b}\right) \frac{1}{|x-s|} d x \\
& \leq C[|\ln \gamma(\tau)|+|\ln h|] h^{2} .
\end{aligned}
$$

By Lemmas 5 and 6, we have

$$
\begin{aligned}
\left|R_{f}(s)\right| & \leq\left|\mathscr{R}^{1}(s)\right|+\left|\mathscr{R}^{2}(s)\right| \\
& \leq C[\eta(s)+|\ln h|+|\ln \gamma(\tau)|] h^{2} .
\end{aligned}
$$

Then the proof is completed.
3.2. The Calculation of $S_{1}(\tau)$. Let $Q_{n}(x)$ be the function of the second kind associated with the Legendre polynomial $P_{n}(x)$, defined by (cf. [24])

$$
Q_{0}(x)=\frac{1}{2} \ln \left|\frac{x+1}{x-1}\right|, \quad Q_{1}(x)=x Q_{0}(x)-1 .
$$

We also define

$$
\begin{array}{r}
W(f, \tau):=f(\tau)+\sum_{i=0}^{\infty}[f(2 i+\tau)+f(-2 i+\tau)], \\
\tau \in(-1,1) .
\end{array}
$$

Then, by the definition of $W$,

$$
\begin{aligned}
W\left(Q_{0}\right)(\tau)= & \frac{1}{2} \ln \frac{1+\tau}{1-\tau} \\
& +\frac{1}{2} \sum_{i=1}^{\infty}\left(\ln \frac{2 i+1+\tau}{2 i-1+\tau}+\ln \frac{2 i-1-\tau}{2 i+1-\tau}\right) \\
= & \frac{1}{2} \lim _{i \rightarrow \infty} \ln \frac{2 i+1+\tau}{2 i+1-\tau}=0, \\
W\left(x Q_{0}^{\prime}\right)(\tau)= & \frac{\tau}{1-\tau^{2}}-\sum_{i=1}^{\infty}\left(\frac{2 i+\tau}{(2 i+\tau)^{2}-1}+\frac{-2 i+\tau}{(-2 i+\tau)^{2}-1}\right) \\
= & \frac{1}{2} \lim _{n \rightarrow \infty} \sum_{k=-n}^{k=n} \frac{1}{k+(1 / 2)-(\tau / 2)}=\frac{\pi}{2} \tan \frac{\pi \tau}{2} .
\end{aligned}
$$

It follows that

$$
S\left(Q_{1}^{\prime}, \tau\right)=W\left(Q_{0}+x Q_{0}^{\prime}, \tau\right)=\frac{\pi}{2} \tan \frac{\tau \pi}{2},
$$

which means

$$
S\left(Q_{1}, \tau\right)=\int \frac{\pi}{2} \tan \frac{\tau \pi}{2} \tau=-\ln \cos \frac{\pi \tau}{2}+C .
$$

What remains is to determine the constant $C$. By using the identities (cf. [24, Chapter 1, Section 1.2]),

$$
\begin{gathered}
x \cot x=1+\sum_{k=1}^{\infty}(-1)^{k} B_{2 k} \frac{(2 x)^{2 k}}{(2 k) !}, \\
\ln (2 \cos x)=-\sum_{j=1}^{\infty} \frac{1}{j} \cos (2 j x), \quad x \in(0, \pi),
\end{gathered}
$$

where $B_{2 k}$ denote the Bernoulli numbers, we have

$$
\begin{aligned}
& \sum_{k=1}^{\infty}(-1)^{k} B_{2 k} \frac{(2 x)^{2 k+1}}{(2 k+1) !} \\
& \quad=2 x \ln (\sin x)+2\left[(\ln 2-1) x+\sum_{j=1}^{\infty} \frac{1}{2 j^{2}} \sin (2 j x)\right] .
\end{aligned}
$$


TABLE 1: Errors of the mod-trapezoidal rule with $s=x_{[0 n / 4]}+(1+\tau) h / 2$.

\begin{tabular}{lcccc}
\hline$n$ & $\tau=0$ & $\tau=2 / 3$ & $\tau=-2 / 3$ & $\tau=1 / 2$ \\
\hline 32 & $3.5392 e-002$ & $-7.1780 e-004$ & $1.1040 e-003$ & $1.7473 e-002$ \\
64 & $1.6961 e-002$ & $-1.9364 e-004$ & $2.7065 e-004$ & $8.4165 e-003$ \\
128 & $8.3004 e-003$ & $-5.0195 e-005$ & $6.6991 e-005$ & $4.1332 e-003$ \\
256 & $4.1057 e-003$ & $-1.2773 e-005$ & $1.6664 e-005$ & $2.0484 e-003$ \\
512 & $2.0417 e-003$ & $-3.2214 e-006$ & $4.1554 e-006$ & $1.0198 e-003$ \\
1024 & $1.0181 e-003$ & $-8.0885 e-007$ & $1.0375 e-006$ & $5.0878 e-004$ \\
\hline$h^{\alpha}$ & 1.0239 & 1.9587 & 2.0111 & 1.0204 \\
\hline
\end{tabular}

TABLE 2: Errors of the mod-trapezoidal rule with $s=x_{0 n}-(1+\tau) h / 2$.

\begin{tabular}{lcccc}
\hline$n$ & $\tau=0$ & $\tau=2 / 3$ & $\tau=-2 / 3$ & $\tau=1 / 2$ \\
\hline 32 & $2.5227 e-002$ & $2.9756 e-002$ & $1.2230 e-001$ & $-8.0605 e-002$ \\
64 & $1.1997 e-002$ & $1.4808 e-002$ & $6.0946 e-002$ & $-4.1289 e-002$ \\
128 & $5.8224 e-003$ & $7.3643 e-003$ & $3.0401 e-002$ & $-2.0913 e-002$ \\
256 & $2.8619 e-003$ & $3.6669 e-003$ & $1.5177 e-002$ & $-1.0529 e-002$ \\
512 & $1.4173 e-003$ & $1.8283 e-003$ & $7.5815 e-003$ & $-5.2839 e-003$ \\
1024 & $7.0490 e-004$ & $9.1253 e-004$ & $3.7886 e-003$ & $-2.6471 e-003$ \\
\hline$h^{\alpha}$ & 1.0323 & 1.0054 & 1.0025 & 0.9857 \\
\hline
\end{tabular}

Setting $x=\pi / 2$ gives

$$
\sum_{k=1}^{\infty}(-1)^{k} B_{2 k} \frac{(\pi)^{2 k+1}}{(2 k+1) !}=\ln 2-1
$$

Then we have

$$
\begin{aligned}
W\left(Q_{1} ; 0\right) & =-1+2 \sum_{i=1}^{\infty} Q_{1}(2 i) \\
& =-1+2 \sum_{i=1}^{\infty} \sum_{k=1}^{\infty} \frac{1}{(2 k+1)(2 i)^{2 k}} \\
& =-1+\sum_{k=1}^{\infty}(-1)^{k+1} B_{2 k} \frac{(\pi)^{2 k+1}}{(2 k+1) !}=-\ln 2,
\end{aligned}
$$

where we have used the formulae (cf. [24, Chapter 1, Section $1.2])$

$$
\begin{gathered}
Q_{1}(x)=\sum_{k=1}^{\infty} \frac{1}{(2 k+1) x^{2 k}}, \quad|x|>1, \\
\sum_{i=1}^{\infty} \frac{1}{i^{2 k}}=\frac{(-1)^{k+1} 2^{2 k-1}}{(2 k) !} B_{2 k} \pi^{2 k} .
\end{gathered}
$$

Then we have

$$
S_{1}(\tau)=-\ln 2 \cos \frac{\tau \pi}{2}
$$

By Theorem 2, we get the following error expansion:

$$
E_{n}(f)=\frac{f^{\prime \prime}(s) h}{2} \log 2 \cos \frac{\pi \tau}{2}+O\left(h^{2}\right) .
$$

Let

$$
T\left(h_{l}\right)=I_{2^{l-1} n_{0}}(f, s), \quad l=1,2, \ldots,
$$

where $n_{0}$ is the starting meshes, $l$ is the refining numbers, and $h_{l}=(b-a) / 2^{l-1} n_{0}$. Then we have the following.

Corollary 7. Under the same assumption of Theorem 2 and (62), there holds

$$
T\left(h_{l}\right)-T\left(h_{l-1}\right)=O\left(h_{l-1}^{2}\right) .
$$

\section{Numerical Examples}

In this section, computational results are reported to confirm our theoretical analysis.

Example 1. Consider the hypersingular integral

$$
f_{0}^{1} \frac{x^{3}}{(x-s)^{2}} d x=3 s+\frac{3}{2}+\frac{1}{s-1}+3 s^{2} \log \frac{1-s}{s} .
$$

We examine the dynamic point $s=x_{[0 n / 4]}+(1+\tau) h / 2$, in Table 1 show that when the local coordinate of singular point $\tau= \pm 2 / 3$, the quadrature reach the convergence rate of $O\left(h^{2}\right)$ as for the nonsupersingular point, there are no convergence rate which agree with our theorematically analysis. For the case of $s=b-(\tau+1) h / 2$, Table 2 shows that there is no superconvergence phenomenon because of the influence of $\eta(s)$ which coincides with our theoretical analysis.

Example 2. Consider the hypersingular integral

$$
f_{0}^{1} \frac{x^{4}+1}{(x-s)^{2}} d x=4 s^{2}+2 s+\frac{4}{3}+\frac{s+1}{s(s-1)}+4 s^{3} \log \frac{1-s}{s} .
$$


TABLE 3: Errors of the mod-trapezoidal rule.

\begin{tabular}{lccccc}
\hline$n$ & $s=1 / 4$ & $h^{\alpha}$ & $s=1 / 2$ & $h^{\alpha}$ & $s=5 / 8$ \\
\hline 32 & $-2.1121 e-003$ & & $-1.8532 e-003$ & & $-1.0260 e-003$ \\
64 & $-5.3080 e-004$ & 1.9925 & $-4.6835 e-004$ & 1.9844 & $-2.6489 e-004$ \\
128 & $-1.3304 e-004$ & 1.9963 & $-1.1770 e-004$ & 1.9924 & $-6.7234 e-005$ \\
256 & $-3.3301 e-005$ & 1.9982 & $-2.9502 e-005$ & 1.9963 & $-1.6933 e-005$ \\
512 & $-8.3306 e-006$ & 1.9991 & $-7.3850 e-006$ & 1.9982 & $-4.2487 e-006$ \\
1024 & $-2.0833 e-006$ & 1.9996 & $-1.8474 e-006$ & 1.9991 & $-1.0641 e-006$ \\
\hline
\end{tabular}

TABLE 4: A posteriori error estimate of the mod-trapezoidal rule.

\begin{tabular}{lccccc}
\hline$n$ & $s=1 / 4$ & $h^{\alpha}$ & $s=1 / 2$ & $h^{\alpha}$ & $s=5 / 8$ \\
\hline 32 & & & & $h^{\alpha}$ \\
64 & $-1.5814 e-003$ & & $-1.3849 e-003$ & & $-7.6112 e-004$ \\
128 & $-3.9776 e-004$ & 1.9912 & $-3.5065 e-004$ & 1.9817 & $-1.9765 e-004$ \\
256 & $-9.9737 e-005$ & 1.9957 & $-8.8202 e-005$ & 1.9911 & $-5.0301 e-005$ \\
512 & $-2.4971 e-005$ & 1.9979 & $-2.2117 e-005$ & 1.9956 & $-1.2684 e-005$ \\
1024 & $-6.2473 e-006$ & 1.9989 & $-5.5375 e-006$ & 1.9978 & $-3.1846 e-006$ \\
\hline
\end{tabular}

TABLE 5: Errors of the mod-trapezoidal rule.

\begin{tabular}{|c|c|c|c|c|c|c|}
\hline$n$ & $i=-1$ & $h^{\alpha}$ & $i=0$ & $h^{\alpha}$ & $i=1$ & $h^{\alpha}$ \\
\hline 32 & $-1.9220 e-002$ & & $-5.6378 e-002$ & & 1.1169 & \\
\hline 64 & $-4.9807 e-003$ & 1.9481 & $-2.1100 e-002$ & 1.4179 & $7.8885 e-001$ & $5.0167 e-001$ \\
\hline 128 & $-1.2750 e-003$ & 1.9659 & $-7.7489 e-003$ & 1.4451 & $5.5758 e-001$ & $5.0058 e-001$ \\
\hline 256 & $-3.2386 e-004$ & 1.9770 & $-2.8116 e-003$ & 1.4626 & $3.9421 e-001$ & $5.0021 e-001$ \\
\hline 512 & $-8.1850 e-005$ & 1.9843 & $-1.0120 e-003$ & 1.4742 & $2.7874 e-001$ & $5.0007 e-001$ \\
\hline 1024 & $-2.0617 e-005$ & 1.9892 & $-3.6228 e-004$ & 1.4820 & $1.9709 e-001$ & $5.0003 e-001$ \\
\hline
\end{tabular}

TABLE 6: A posteriori error estimate of the mod-trapezoidal rule.

\begin{tabular}{|c|c|c|c|c|c|c|}
\hline$n$ & $i=-1$ & $h^{\alpha}$ & $i=0$ & $h^{\alpha}$ & $i=1$ & $h^{\alpha}$ \\
\hline \multicolumn{7}{|l|}{32} \\
\hline 64 & $-1.4239 e-002$ & & $-3.5278 e-002$ & & $3.2804 e-001$ & \\
\hline 128 & $-3.7057 e-003$ & 1.9420 & $-1.3351 e-002$ & 1.4019 & $2.3128 e-001$ & $5.0427 e-001$ \\
\hline 256 & $-9.5112 e-004$ & 1.9621 & $-4.9373 e-003$ & 1.4351 & $1.6337 e-001$ & $5.0150 e-001$ \\
\hline 512 & $-2.4201 e-004$ & 1.9746 & $-1.7996 e-003$ & 1.4560 & $1.1548 e-001$ & $5.0053 e-001$ \\
\hline 1024 & $-6.1233 e-005$ & 1.9827 & $-6.4972 e-004$ & 1.4698 & $8.1643 e-002$ & $5.0019 e-001$ \\
\hline
\end{tabular}

The numerical results show that the convergence rate reaches $\left(h^{2}\right)$ when the singular point coincides with the mesh point in Table 3. In Table 4, a posteriori error estimate is presented and the convergence rate is also $O\left(h^{2}\right)$ which agrees with our theoretical analysis.

Example 3. Now we consider an example of less regularity. Let $a=-b=-1, s=0$, and

$$
\begin{array}{r}
f(x)=\mathscr{F}_{i}(x):=x^{2}+(2+\operatorname{sign}(x))|x|^{2-i+0.5}, \\
i=-1,0,1 .
\end{array}
$$

Obviously, $\mathscr{F}_{i}(x) \in C^{3-i+0.5}[-1,1](i=-1,0,1)$. The exact value of the integral is

$$
\mathscr{I}_{2}\left(\mathscr{F}_{i}(x), 0\right)=\frac{14-4 i}{3-2 i}
$$

The numerical results are presented in Tables 5 and 6 . When the density function $f(x)$ is smooth enough $i=$ -1 , the error bound is $O\left(h^{2}\right)$, and if the density function has less regularity $i=0,1$, there is no hyperconvergence phenomenon, which means the regularity of density function cannot be reduced.

\section{Acknowledgments}

The work of Jin Li was supported by the National Natural Science Foundation of China (nos. 11101247, 11201209, and 11101246), China Postdoctoral Science Foundation Fund Project (no. 2013M540541), the Shandong Provincial Natural Science Foundation of China (no. ZR2011AQ020), and a Project of Shandong Province Higher Educational Science and Technology Program (no. J11LE8). 


\section{References}

[1] G. Monegato, "Numerical evaluation of hypersingular integrals," Journal of Computational and Applied Mathematics, vol. 50, no. 1-3, pp. 9-31, 1994.

[2] U. J. Choi, S. W. Kim, and B. I. Yun, "Improvement of the asymptotic behaviour of the Euler-Maclaurin formula for Cauchy principal value and Hadamard finite-part integrals," International Journal for Numerical Methods in Engineering, vol. 61, no. 4, pp. 496-513, 2004.

[3] J. T. Chen and H. K. Hong, "Review of dual boundary element methods with emphasis on hypersingular integrals and divergent series," Applied Mechanics Reviews, vol. 52, no. 1, pp. 17-32, 1999.

[4] A. Frangi and M. Guiggiani, "A direct approach for boundary integral equations with high-order singularities," International Journal for Numerical Methods in Engineering, vol. 49, no. 7, pp. 871-898, 2000.

[5] T. Hasegawa, "Uniform approximations to finite Hilbert transform and its derivative," Journal of Computational and Applied Mathematics, vol. 163, no. 1, pp. 127-138, 2004.

[6] C. Y. Hui and D. Shia, "Evaluations of hypersingular integrals using Gaussian quadrature," International Journal for Numerical Methods in Engineering, vol. 44, no. 2, pp. 205-214, 1999.

[7] N. I. Ioakimidis, "On the uniform convergence of Gaussian quadrature rules for Cauchy principal value integrals and their derivatives," Mathematics of Computation, vol. 44, no. 169, pp. 191-198, 1985.

[8] P. Kim and U. J. Choi, "Two trigonometric quadrature formulae for evaluating hypersingular integrals," International Journal for Numerical Methods in Engineering, vol. 56, no. 3, pp. 469-486, 2003.

[9] J. Li, J. M. Wu, and D. H. Yu, "Generalized extrapolation for computation of hypersingular integrals in boundary element methods," Computer Modeling in Engineering \& Sciences, vol. 42, no. 2, pp. 151-175, 2009.

[10] J. Li and D. H. Yu, "The superconvergence of certain twodimensional Cauchy principal value integrals," Computer Modeling in Engineering \& Sciences, vol. 71, no. 4, pp. 331-346, 2011.

[11] J. Li and D. H. Yu, "The superconvergence of certain twodimensional Hilbert singular integrals," Computer Modeling in Engineering \& Sciences, vol. 82, no. 3-4, pp. 233-252, 2011.

[12] J. Li and D. H. Yu, "The error estimate of Newton-Cotes methods to compute hypersingular integrals," Mathematica Numerica Sinica, vol. 33, no. 1, pp. 77-86, 2011.

[13] J. Li, X. Zhang, and D. Yu, "Superconvergence and ultraconvergence of Newton-Cotes rules for supersingular integrals," Journal of Computational and Applied Mathematics, vol. 233, no. 11, pp. 2841-2854, 2010.

[14] J. Li, X. Zhang, and D. Yu, "Extrapolation methods to compute hypersingular integral in boundary element methods," Science China Mathematics, vol. 56, no. 8, pp. 1647-1660, 2013.

[15] M. N. J. Moore, L. J. Gray, and T. Kaplan, "Evaluation of supersingular integrals: second-order boundary derivatives," International Journal for Numerical Methods in Engineering, vol. 69, no. 9, pp. 1930-1947, 2007.

[16] Q. K. Du, "Evaluations of certain hypersingular integrals on interval," International Journal for Numerical Methods in Engineering, vol. 51, no. 10, pp. 1195-1210, 2001.

[17] J. Wu and W. Sun, "The superconvergence of the composite trapezoidal rule for Hadamard finite part integrals," Numerische Mathematik, vol. 102, no. 2, pp. 343-363, 2005.
[18] X. Zhang, J. Wu, and D. Yu, "Superconvergence of the composite Simpson's rule for a certain finite-part integral and its applications," Journal of Computational and Applied Mathematics, vol. 223, no. 2, pp. 598-613, 2009.

[19] D. H. Yu, Natural Boundary Integral Method and Its Applications, Kluwer Academic Publishers, 2002.

[20] P. Linz, "On the approximate computation of certain strongly singular integrals," Computing, vol. 35, no. 3-4, pp. 345-353, 1985.

[21] D. H. Yu, "The approximate computation of hypersingular integrals on interval," Numerical Mathematics, vol. 1, no. 1, pp. 114-127, 1992.

[22] J. M. Wu and D. H. Yu, "An approximate computation of hypersingular integrals on an interval," Journal on Numerical Methods and Computer Applications, vol. 19, no. 2, pp. 118-126, 1998.

[23] J. Wu, Y. Wang, W. Li, and W. Sun, "Toeplitz-type approximations to the Hadamard integral operator and their applications to electromagnetic cavity problems," Applied Numerical Mathematics, vol. 58, no. 2, pp. 101-121, 2008.

[24] L. C. Andrews, Special Functions of Mathematics for Engineers, McGraw-Hill, New York, NY, USA, 2nd edition, 1992. 


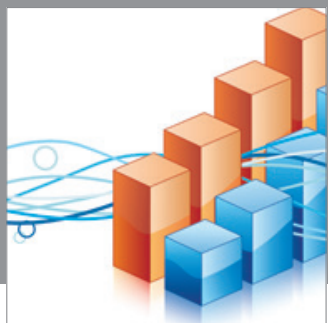

Advances in

Operations Research

mansans

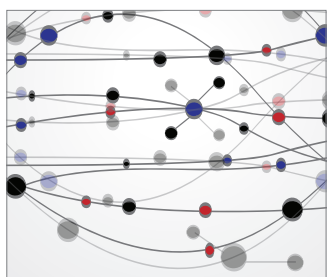

The Scientific World Journal
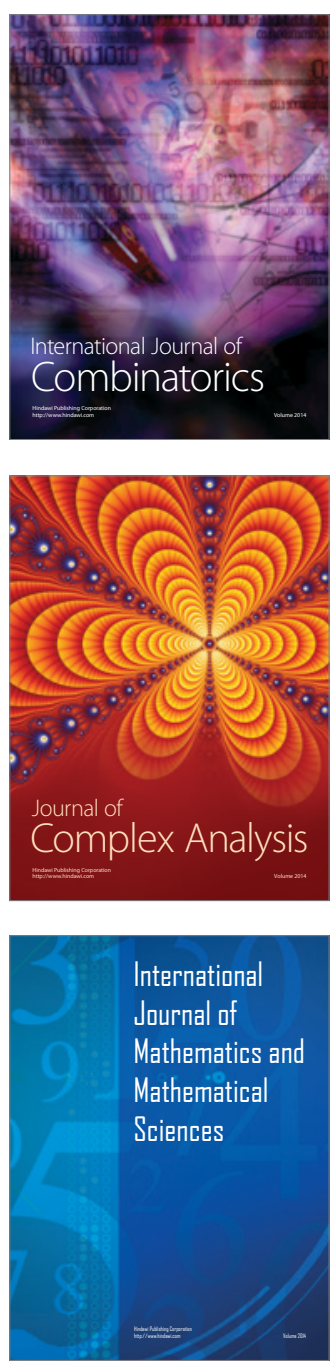
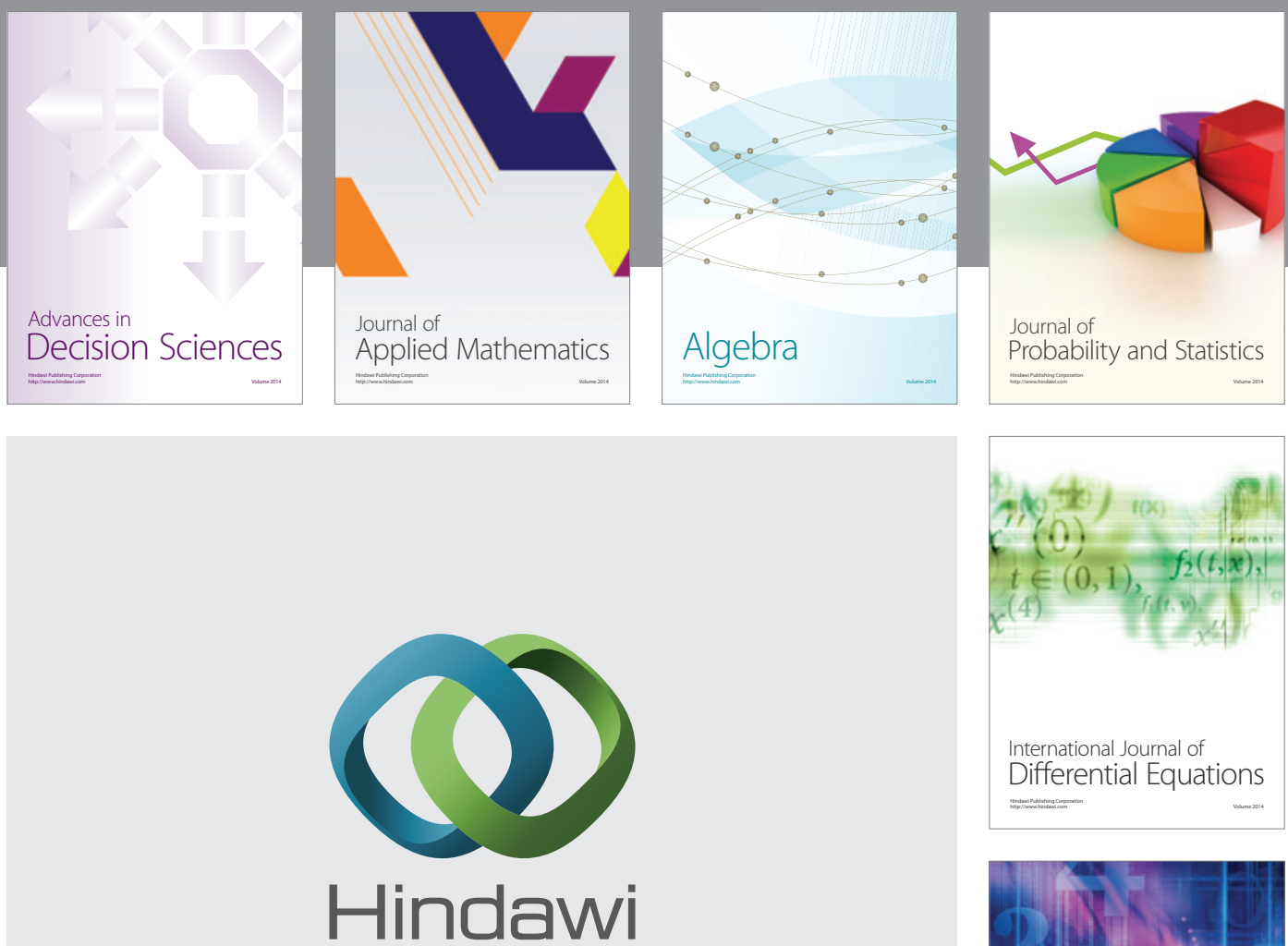

Submit your manuscripts at http://www.hindawi.com
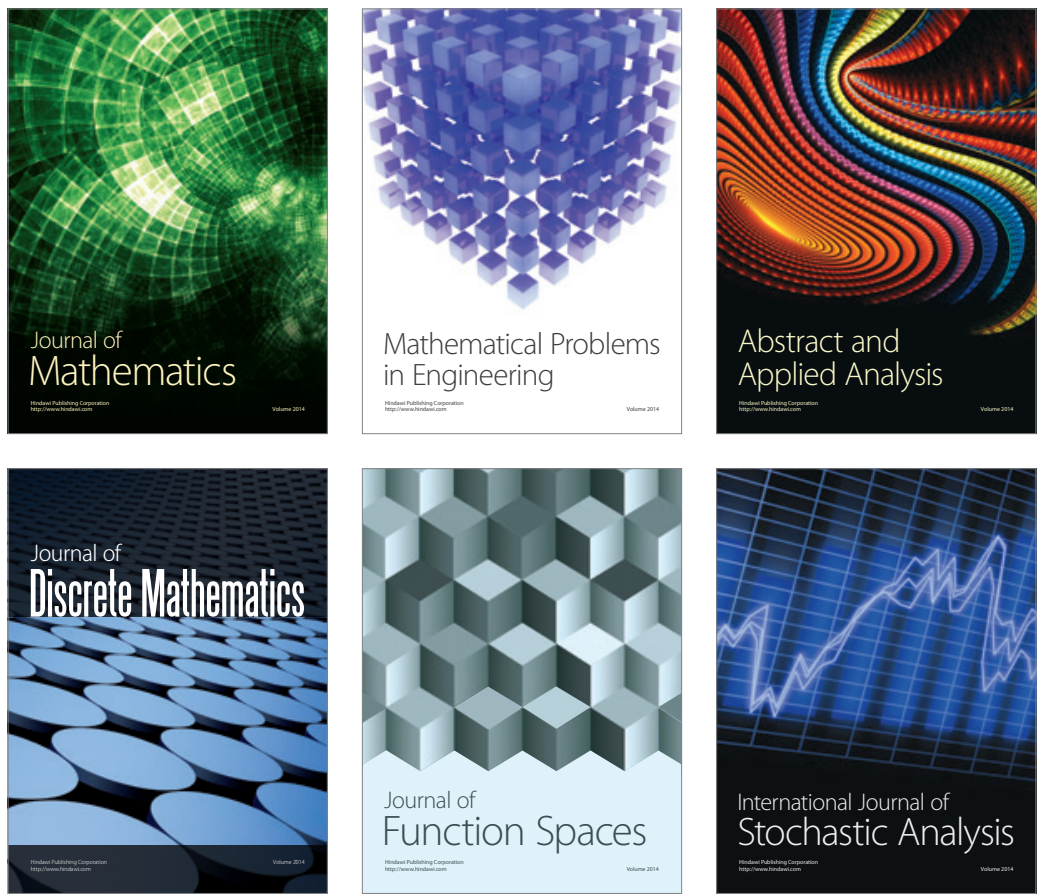

Journal of

Function Spaces

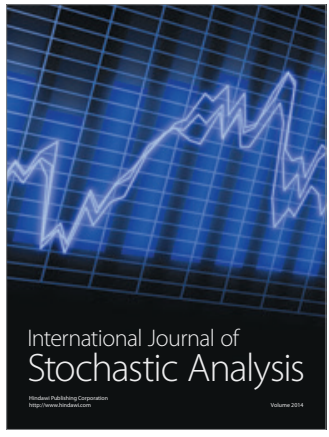

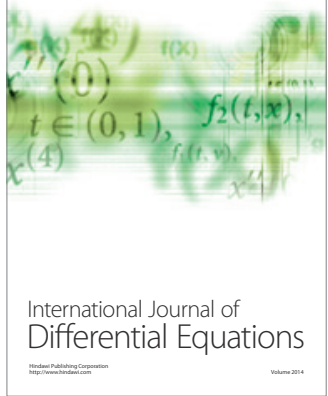
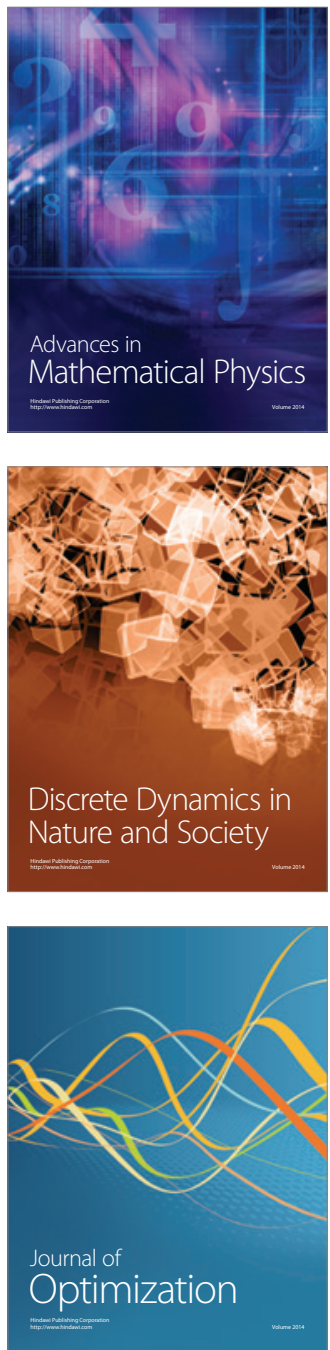